Vol. 69, N. ${ }^{\circ}$ IIO (noviembre), 4I-5I

\title{
LA ORGANIZACIÓN COMUNITARIA: UN ELEMENTO CLAVE DEL CAPITAL SOCIAL PARA EL DESARROLLO ECONÓMICO Y SOCIAL DE LA PARROQUIA DE CANGAHUA
}

\author{
IRMA GARCÍA-SERRANO, RAMIRO VILLARRUEL-MEYTHALER \\ Universidad Central del Ecuador
}

Recepción de manuscrito: 11 de septiembre de 2017 Aceptación del manuscrito: 23 de octubre de 2017

\begin{abstract}
RESUMEN En este artículo se analiza a la organización comunitaria como un elemento importante del capital social. Se evidencia una influencia directa del capital social en el desarrollo económico y social de la comunidad Compañía Lote Dos en la parroquia de Cangahua del cantón Cayambe (Ecuador). Para observar el efecto que tiene la organización comunitaria en el bienestar socioeconómico de la comunidad, se desarrolla un modelo econométrico de regresión con variables dicotómicas (ANOVA) que muestra cómo el pertenecer a una organización comunitaria mejora las condiciones de vida representadas por las variables ingreso, escolaridad y producción agrícola de las familias de la comunidad.
\end{abstract}

PALABRAS ClAVE Organización comunitaria, capital social, desarrollo socioeconómico, modelos econométricos.

ABSTRACT In this article the community organization is analyzed as an important element of social capital. The social capital shows a direct influence on economic and social of the community Compañía Lote Dos of Cangahua, Cayambe (Ecuador). In order to observe the effect that the community organization has on this socioeconomic well-being of the community, an econometric model of regression with dichotomous variables (ANOVA), is developed, which shows how belonging to a community organization improves the living conditions, which are represented by the variables income, schooling and agricultural production.

KEYWORDS Community organization, social capital, socioeconomic development, econometric models.

Jel CODES C51, R11, Z13.

\section{INTRODUCCIÓN}

La participación de los individuos en los asuntos que atañen a su vida, sea de forma individual o colectiva, así como la decisión de ser parte de una organización, se convierten en un recurso que disponen las familias para enfrentar y buscar la solución a sus problemas. Pertenecer a una organización comunitaria desarrolla relaciones de cooperación (Lesser, 2000; Fukuyama, 2001), confianza, comprensión, tolerancia y reciprocidad (Campbell y Jovchelovitch, 200o). La organización permite mejorar las oportunidades para gestionar, demandar y negociar con otras organizaciones, gobierno local, estado, entre otros con el propósito de conseguir objetivos para 
su propio desarrollo (Food and Agriculture Organization [FAO], 2008). En el ámbito del desarrollo comunitario, Putnam (2001) establece una relación entre capital social y el ámbito local comunitario, y plantea una visión sociocomunitaria cuyo propósito es promover los objetivos económicos o sociales de la población (Carpio, 2001; Gutiérrez 2016). Asimismo, Matus (1986) y Falleto y Martner (1986) mencionan que las organizaciones comunitarias deben construir viabilidad colaborativa para fortalecer su desarrollo; otros, por su parte, afirman que el trabajo en redes es un elemento esencial en el desarrollo social (Schiff, 1992; Stone, 2000; Delhey y Newton, 2002; Roche, 2004; Van Deth, 2008) y económico (Mavares et al., 1999; Ibañez et al., 2004; Martínez, 2010; Sánchez, 2013).

Es así como el capital social se entiende como el conjunto de relaciones sociales basadas en la confianza, en comportamientos de cooperación y reciprocidad. Además, se enmarca en el ámbito del desarrollo que permite a las comunidades lograr un cierto nivel de crecimiento. Igualmente, el capital social promueve la participación en un nivel comunitario asociativo como una oportunidad para articular algunos servicios públicos en beneficio de los hogares, lo que es muy importante en programas orientados a reducir la pobreza (Atria, Siles, Arriagada, Robison y Whiteford, 2003).

La hipótesis central de este estudio sugiere que las familias que conforman una organización comunitaria adquieren un mayor capital social y, a su vez, esto les permite disponer de un mayor nivel de ingreso, una mayor escolaridad y una mayor producción agrícola. Sin embargo, el capital social varía de acuerdo con el grado de involucramiento y de pertenencia que los individuos van adquiriendo en la organización de su comunidad.

Las familias objeto de este estudio se han localizado en la comunidad Compañía Lote Dos de la parroquia de Cangahua en el cantón Cayambe de Ecuador. Fue establecida en 1960, toma su nombre de la Hacienda la Compañía, en la que trabajaban los huasipungueros que posteriormente formaron la comuna. Para la investigación se utilizó el censo sociodemográfico y productivo levantado en la comunidad, mismo que permitió la medición de la brecha que existe entre las familias que conforman o no una organización comunitaria en cuanto al ingreso, escolaridad y producción agrícola a través de la técnica econométrica de regresión ANOvA.

Investigaciones empíricas como las citadas sustentan que la organización comunitaria beneficia al desarrollo social y económico de sus miembros. Pese a ello, estos estudios no han analizado cuantitativamente la brecha en cuanto a los ingresos, la escolaridad y la producción agrícola, entre las familias que pertenecen o no a una organización comunitaria tal como se muestra en esta investigación.

Los resultados de esta investigación sugieren que la pertenencia a una organización comunitaria, elemento clave del capital social, les permite a las familias de la comunidad Compañía Lote Dos, Cangahua-Cayambe, alcanzar un mayor ingreso, presentar un mayor nivel de escolaridad y una mayor productividad agrícola concordando con las mencionadas investigaciones de Matus (1986), Falleto y Martner (1986), Putnam (2001), entre otros sobre el valor que el capital social tiene para producir beneficios económicos y contribuir a reducir la pobreza.

El artículo se divide en seis partes, incluida esta introducción. Primero se realiza un análisis sobre la conceptualización del capital social. Posteriormente se describe cómo la organización comunitaria representa un elemento del capital social que genera efectos positivos en 
el crecimiento socioeconómico de los integrantes de una comunidad. Luego de este análisis se describe la metodología de la investigación. En la quinta sección, se presentan los resultados de la investigación con el uso del modelo econométrico de regresión y, finalmente, se exponen las conclusiones referentes a la investigación.

\section{MARCO TEÓRICO}

El fundamento teórico de este artículo es el capital social, término cuyo uso se ha incrementado tanto en el círculo académico como en organizaciones que trabajan por el desarrollo debido a que el capital social integra otros conceptos de diferentes campos como la reciprocidad, las redes sociales, el desarrollo comunitario, la participación y la gobernabilidad, entre los más importantes, Durston (2000, p. 103-108). Si se parte del origen de la variable capital social, pareciera que la idea surgió de Coleman (Ramírez, 2005, p. 21), quien utilizó el concepto para describir un recurso intangible que emerge de sus lazos sociales. Otro estudio, como el descrito por Fernández (2012), menciona a Bourdieu como el autor de las primeras ideas del término capital social, que lo usó para referirse a los beneficios que obtienen las personas al ser miembros de ciertas comunidades.

Según Bourdieu (1997), el capital social es el resultado de una gestión exitosa del capital colectivamente adquirido por los individuos. Éste está representado por un conjunto de recursos movilizados a través de una red de relaciones más o menos extensas, llegando a afirmar que las ganancias obtenidas debido a la pertenencia a un grupo son la base de la solidaridad (Bourdieu, 1985, p. 249).

Continúa explicando que las familias están sometidas a dos sistemas de fuerzas contradictorias. Por una parte, las fuerzas de la economía y, por otra, las fuerzas de la cohesión, que en cierto modo se refieren al hecho de que la reproducción del capital, bajo sus diferentes formas, depende en gran medida de la reproducción de la unidad familiar. De esta manera, Bourdieu (1997) puntualiza que el capital simbólico y el capital social solo pueden reproducirse dentro de la unidad social elemental que es la familia.

Una segunda fuente contemporánea es la obra de Loury (1977), quien realiza su análisis en el contexto de su crítica a las teorías neoclásicas sobre la desigualdad racial de los ingresos y sus implicaciones políticas. Sostiene que las teorías económicas ortodoxas eran demasiado individualistas, ya que se centran exclusivamente en el capital humano individual. En su definición, Loury capta las diferencias de acceso a las oportunidades a través de las conexiones sociales para los jóvenes pertenecientes a sectores minoritarios y no minoritarios. Esta obra allanó el camino para el análisis más preciso de Coleman al comentar el rol que juega el capital social en la creación del capital humano y, a su vez, el autor comparte que el capital social trata ciertos aspectos de las relaciones sociales que constituyen recursos intangibles e inherentes a éstas y que pueden ser apropiados o usados por los actores comunitarios en su beneficio (Coleman, 1988).

Además de los aportes teóricos de Bourdieu, Loury y Coleman, se han publicado otros análisis del capital social. Uno de ellos ha sido Baker (1990), quien lo definió como un recurso que los actores sacan de estructuras sociales específicas y luego la emplean en la búsqueda de 
sus beneficios. Esto se genera debido a cambios en la relación entre los actores (Baker, 1990, p. 619). En términos más frecuentes, Schiff (1992, p. 161) define al capital social como el conjunto de elementos de la estructura social que afectan a las relaciones entre las personas. Así también, Woolcock (1997) plantea que el capital social se refiere a las normas y redes que facilitan la acción colectiva. Del mismo modo, Putnam (1993) define el capital social como aquellos aspectos de la organización social tales como la confianza, las normas y las redes que pueden mejorar la eficiencia de la sociedad.

Es importante observar como Loury y Coleman hacen énfasis en la densidad de las redes como condición para el surgimiento del capital social (Portes, 1999, p. 6). Además, es necesario mencionar que tanto Coleman como Bourdieu y Putnam hacen extensas referencias del capital social como un atributo de los grupos sociales, colectividades y comunidades. También analizan el rol de las instituciones sociales en la creación del mismo; del mismo modo se puede aclarar que el capital social es más una propiedad de los grupos que una propiedad de los individuos (Portela y Neira, 2002, p. 28).

En este contexto, una persona, a más de un capital económico, dispone de otros capitales como el cultural fruto de su entorno y sus relaciones. Pese a ello, tanto el capital social individual como el capital social comunitario forman parte de la cultura compartida por las personas que forman una comunidad.

LA ORGANIZACIÓN COMUNITARIA: UNA APROXIMACIÓN AL CAPITAL SOCIAL

El capital social comunitario es la institucionalidad formal e informal que integra las pautas culturales de confianza y cooperación entre todos los sujetos de un sistema social. Una de las características del capital social comunitario es el relacionamiento entre las personas u organizaciones del mismo entorno. Además, procura mejorar las relaciones horizontales entre las organizaciones y los grupos que interactúan en una comunidad (Forni, Castronuovo y Nardone, 2009).

Existen diferentes manifestaciones de cómo el capital social puede influir en la organización comunitaria. Justamente la organización y la presencia de redes, sean éstas formales o informales, generan potencialidades individuales y colectivas en beneficio de toda la comunidad (Putnam, 1993). Asimismo, se puede mencionar que el capital social varía conforme al entorno de cada comunidad o sociedad; es decir, no es el mismo capital social, humano o físico en una comunidad eminentemente rural y agraria que en una sociedad donde prima el comercio y los servicios.

La aplicación del capital social en una comunidad puede generar algunos activos como la coordinación de tareas para conseguir algún objetivo, la participación y el consenso en la toma de decisiones colectivas, el manejo transparente de los recursos, la socialización de la información, entre otros. La existencia del capital social provoca que las acciones se realicen con mayor efectividad, ya que se ejecutan basadas en la confianza y la reciprocidad entre los miembros de la comunidad. Así, este esquema contradice la lógica del mercado que busca la maximización del beneficio individual en el intercambio. Una aproximación empírica del término capital social se logra vinculando este concepto con la variable nivel de asociacionismo o nivel de participación de las familias en organizaciones comunitarias. 
La organización comunitaria, misma que se relaciona con el término capital social, es una variable que afecta de manera positiva al desarrollo socioeconómico de los individuos que conforman una comunidad. Es así como Temple (2001) en su estudio muestra que una de las variables que generan un aumento de la educación y, por ende, del capital social es la participación política y comunitaria de las personas. Asimismo, Nie, Junn and Stehlik-Barry (1996) mencionan que la educación de los individuos crece si existe por parte de ellos un gran compromiso político y social, características claves de la organización comunitaria.

Por su parte, Durston (1999) indica que la participación a nivel comunitario asociativo puede ser clave para articular los servicios públicos con el individuo o el hogar. Por lo tanto, esto permite la superación de los índices de pobreza de las personas u hogares. Esta descripción coincide con el estudio de Forni, Castronuovo, y Nardone (2009) en el que se explica cómo las organizaciones de base o comunitarias han permitido luchar en ámbitos de exclusión social y pobreza.

\section{METODOLOGÍA}

Esta investigación es de tipo cuantitativa, ya que requiere de la aplicación de modelos econométricos que permitan medir la brecha que existe entre las familias que pertenecen a una organización comunitaria y las familias que no. Esta brecha ha diferenciado a las familias de acuerdo con ciertos indicadores tales como el nivel de ingresos de las familias, la escolaridad del jefe o de la jefa del hogar y la incidencia de la pobreza.

Una regresión calcula, comúnmente, la influencia de variables independientes o regresoras cuantitativas sobre una variable dependiente o regresada. Pese a ello, existen modelos de regresión que incluyen variables independientes cualitativas (Wooldridge, 2010, p. 225). Uno de éstos es el modelo con variables dicótomas ANOvA - análisis de varianza-, el cual procura evaluar la significancia estadística de la relación entre una variable regresada cuantitativa y las regresoras cualitativas o dicótomas. Asimismo, este modelo mide la brecha entre los valores medios de dos o más categorías de una variable cualitativa en función a una variable cuantitativa (Gujarati y Porter, 2010, p. 50-87).

Las variables cualitativas denotan, en cualquier modelo de regresión, la presencia o ausencia de una característica o atributo en un determinado grupo poblacional o zona geográfica. Para este estudio, los grupos poblacionales representan las familias que pertenecen o no a una organización comunitaria en la zona de la Compañía Lote Dos, Cangagua-Cayambe, provincia de Pichincha.

Con base en la teoría estadística, las variables de tipo cualitativo muestran información que no puede cuantificarse (Gujarati y Porter, 2010, p. 50-87). Es así como para medir la brecha antes mencionada se ha generado una variable dicotómica artificial, la misma que toma valores de o o 1 . Donde el valor de 1 indica la presencia del atributo, es decir, las familias que pertenecen a una organización comunitaria, mientras que el o indica la ausencia de dicho atributo — familias que no pertenecen a una organización comunitaria-. Por tanto, la utilización de un modelo ANOvA, calculado a través del método de los mínimos cuadrados ordinarios (MCO), ha sido la principal metodología de trabajo para medir la brecha que existe en 
el ingreso, la escolaridad y la pobreza entre las familias que pertenecen a una organización comunitaria y las familias que no.

Siguiendo con la especificación econométrica del modelo de regresión, las ecuaciones han sido definidas así:

$$
\mathrm{Yi}=\hat{\beta}_{1}+\hat{\beta}_{2} \mathrm{X}_{\mathrm{i}}+\hat{\mathrm{u}}_{\mathrm{t}}
$$

Donde:

$Y$ representa a cada variable dependiente cuantitativa -ingresos de las familias, escolaridad del jefe o jefa del hogar y producción agrícola de las familias-.

$X$ representa la variable independiente cualitativa dicotómica con información de las familias que pertenecen a una organización comunitaria o no.

ût es el error de estimación estocástico.

$\hat{\beta}$ es el intercepto de la regresión.

$\hat{\beta}_{2}$ es la pendiente de la regresión.

Una vez estimados los modelos de regresión, esta investigación comprobaría si efectivamente el hecho de que una familia pertenezca a una organización comunitaria mostraría un mayor nivel de ingresos, escolaridad y productividad agrícola en comparación a las familias que no forman parte de una organización comunitaria. Es importante señalar que para la obtención de las variables que conforman los modelos de regresión se utiliza el Censo de Condiciones de Vida de la comunidad Compañía Lote Dos, el mismo que fue levantado por docentes y estudiantes de la Facultad de Ciencias Económicas de la Universidad Central del Ecuador en el año 2015.

\section{RESULTADOS}

En primer lugar se presentan algunas características de la población objeto del estudio. La comunidad, en el año 2015, estaba conformada por 115 familias y aproximadamente 500 pobladores. Entre los principales productos que se cosechan en esta comunidad se encuentran la papa, el melloco, la mashua, el haba; los mismos que se los utiliza para el consumo interno; mientras que la producción de la cebolla blanca es destinada para la comercialización en el mercado. ${ }^{1}$

En lo que respecta al sexo, el 55,65\% de la población censada son mujeres y el restante porcentaje, 44,35\%, son hombres. El grupo de edad con mayor participación es el constituido por personas menores de 18 años, con un 47,49\%, seguido por el grupo comprendido de personas entre 19 y 30 años con un $21,76 \%$, continúa el grupo de 31 a 40 años y, finalmente los grupos menos representados son los que tienen más de 51 años y entre 41 y 50 años con el $8,79 \%$ y $7,53 \%$ respectivamente. En cuanto al nivel de educación de los jefes y jefas de hogar de la población, los que no tienen ninguna formación alcanzan un $67,35 \%$, le sigue la población con un nivel primario que representa un 29,08\%, seguido por la población con un nivel secundario con el 3,06 y, por último, la población que tiene formación universitaria representa el $0,51 \%$. 
Tabla 1. Resultados del modelo econométrico de regresión ANOVA

\begin{tabular}{lccc}
\hline \multicolumn{1}{c}{ Indicador } & $\begin{array}{r}\text { Jefe o jefa de hogar que } \\
\text { pertenece a una }\end{array}$ & $\begin{array}{c}\text { Jefe o jefa de hogar que no } \\
\text { pertenece a una } \\
\text { organización comunitaria }\end{array}$ & organización comunitaria \\
\hline Ingreso del hogar (USD) & 203,94 & 163,86 & 40,08 \\
Escolaridad (años) & 7,30 & 5,33 & 1,97 \\
Producción agrícola (USD) & 374,32 & 208,64 & 165,68 \\
\hline
\end{tabular}

Fuente: Censo de Condiciones de vida de la comunidad Compañía Lote Dos de la parroquia de Cangahua.

Así también, de acuerdo con el censo, es importante mencionar que el 40,19\% de los jefes o jefas de hogar pertenecen a alguna organización comunitaria. Entre las organizaciones comunitarias que más miembros de la comunidad Compañía Lote Dos alcanzan, son las organizaciones relacionadas a temas agrícolas y/o ambientales, comerciales, deportivas y socioculturales.

Los indicadores comúnmente usados para medir el desarrollo social y económico de una población son la pobreza y la exclusión social. Éstos se acentúan en mayor magnitud en las comunidades rurales de Ecuador como la comunidad Compañía Lote Dos de la parroquia de Cangahua. La investigación revela que el nivel de ingreso, la escolaridad y la incidencia de la pobreza de esta comunidad se encuentran por debajo de las cifras promedio del país. La incidencia de la pobreza por ingresos a nivel nacional, en el año 2015, se ubicó en 23,3\%, mientras que la comunidad Compañía Lote Dos reflejó una cifra de 56,2\%. En cuanto a la incidencia nacional de la pobreza por necesidades básicas insatisfechas (NBI), ésta fue de 32,9\%; mientras que la comunidad alcanzó una cifra de $88,7 \%$ en el mismo periodo de análisis. En lo que respecta al nivel de escolaridad en el país, presentó un resultado de 10,1 años promedio, cifra superior a la reflejada en la comunidad, que fue 6,85 años. Por último, el promedio de los ingresos mensuales de todos los ecuatorianos en el año 2015 fue USD 469,71, mientras que el ingreso promedio de los miembros de la comunidad Compañía Lote Dos fue USD 167,42. ${ }^{2}$

Después de haber revisado la literatura relevante acerca del capital social y su relación con la organización comunitaria, en este artículo se intenta demostrar que muchos de los elementos citados reflejan el objeto de estudio de esta investigación. Es así como se observa que los hogares de la comunidad que pertenecen a una organización comunitaria adquieren ciertos recursos potenciales para enfrentar problemas socioeconómicos que viven en su cotidianidad. El principal resultado del estudio evidencia cómo los hogares de la comunidad analizada que pertenecen a una organización comunitaria, han alcanzado un mayor ingreso, un mayor nivel de escolaridad y una mayor productividad agrícola. Esto se ha demostrado a través del uso del modelo de regresión Anova (ver Tabla 1).

En primer lugar, el ingreso promedio mensual de las familias que no pertenecen a una organización comunitaria se ubica en USD 163,86; mientras que las familias que pertenecen a dichas organizaciones tienen un ingreso de USD 203,94. Por lo tanto, existe una brecha de USD 40,08 entre estos dos grupos poblacionales para el año 2015. Estos resultados reflejan que las familias que pertenecen a una organización comunitaria disponen de mayores ingresos, los mismos que contribuyen en su desarrollo económico (Mavares et al., 1999; Ibañez et al., 2004; Martínez, 2010; Sánchez, 2013). 
El mismo resultado se ha observado en cuanto a la escolaridad de los jefes y las jefas del hogar. Para aquellos que forman parte de cualquier organización comunitaria, se ha notado una escolaridad promedio de 7,3 años de escolaridad, cifra superior a la escolaridad de jefes y jefas que no pertenecen a dichos grupos que ha sido de 5,3 años de escolaridad. Estos hallazgos coinciden con los estudios de Temple (2001) y Nie, Junn y Stehlik-Barry (1996), quienes puntualizan una relación positiva entre la organización comunitaria y la educación.

Por último, la brecha en cuanto a la producción agrícola entre las familias que se han incluido o no a una organización comunitaria es de USD 165,68; siendo la producción agrícola de las familias que pertenecen a una organización superior a la producción de las familias que no.

Así también se ha comprobado de manera descriptiva que la incidencia de la pobreza por ingresos en las familias que pertenecen a una organización comunitaria, ha sido de 44\%; mientras que la pobreza por NBI para este mismo grupo de familias fue de $84,7 \%$. Por otro lado, la pobreza por ingresos para las familias que no pertenecen a ninguna organización comunitaria ha sido de $63 \%$ y la pobreza por NBI se ubicó en el 93,4\%. Estas cifras reflejan un comportamiento semejante en estudios (Schiff, 1992; Durston, 1999; Stone, 2000; Delhey y Newton, 2002; Atria et al., 2003; Roche, 2004; Van Deth, 2008) en los que se explica que la organización de la comunidad ha permitido reducir los índices de exclusión social y pobreza.

Es preciso mencionar que los modelos ANOva tienen como objetivo medir las diferencias existentes entre dos grupos poblacionales con características opuestas - hogares que pertenecen una organización comunitaria y otros que no pertenecen a la organización- Esta diferencia se representa a través de una variable binaria, siendo la variable independiente cuantitativa -ingreso, escolaridad y producción agrícola-. Es así como el modelo se especifica como el ingreso en función de la variable binaria, la cual, al ser estadísticamente significativa, refleja que existen diferencias entre estos grupos. Algo similar ocurre con los modelos en los que las variables son escolaridad y producción agrícola.

Cabe señalar que en todos estos modelos la variable dependiente es la que cambió, mientras que la variable independiente, pertenece a la organización (1) o no pertenece (o) es la misma en todos los modelos.

En suma, se observa que la variable relacionada a la organización de la comunidad es importante para lograr buenos resultados en la producción agrícola, mejorar los ingresos y aumentar el nivel de escolaridad. La organización de una comunidad es exitosa cuando hay confianza, solidaridad y respeto, es decir, cuando existe capital social como lo manifiesta Aguerre (2011), que explica que la confianza en las redes está dada en la medida que los individuos se identifiquen con ellas.

\section{CONCLUSIONES}

En esta investigación se compara el ingreso, la escolaridad y la productividad agrícola de dos grupos poblacionales. El primero representa a las familias que conforman una organización comunitaria y el segundo constituye a las familias que no. Con los resultados obtenidos, se comprueba que el primer grupo de hogares disponen de un mayor capital social; por tanto, estas cifras concuerdan con la hipótesis planteada para la investigación. 
Pese a que existe una gran brecha entre los dos grupos poblacionales analizados, es claro como estos indicadores sociodemográficos están lejos al promedio nacional. Esto refleja el hecho de que el área rural de Ecuador continúa desatendida por el gobierno central y por los gobiernos seccionales. Por ende, es importante que las localidades rurales ecuatorianas traten de romper el círculo de la pobreza y sus consecuencias a través de la organización comunitaria.

Si se demuestra que la organización, como elemento del capital social, tiene más efectos positivos que negativos, el Estado ecuatoriano debería promover las asociaciones locales, motivar la participación, el voluntariado y el empoderamiento como mecanismos que a más de impulsar el contacto y las relaciones sociales sean el eje para fomentar la cohesión, el compromiso cívico y sobre todo los vínculos comunitarios. Muchas de estas acciones, en varias naciones, han sido responsabilidad de organizaciones no gubernamentales; sin embargo, se debería promover acciones para que los gobiernos locales fomenten que las familias opten por ser parte de instituciones más formales de asociación y participación; por lo que la organización comunitaria debería ser reconocida como tema importante de políticas públicas. La sociedad y el Estado debería analizar los efectos positivos del capital social y facilitar el acceso de la gente pobre para acrecentar su capital social y lograr mejores resultados no solo económicos, sino también sociales, científicos, culturales, entre otros.

\section{NOTAS}

1 Las cifras de los indicadores socioeconómicos a nivel nacional se calcularon de la Encuesta Nacional de Empleo, Desempleo y Subempleo (ENEMdu) levantada por el Instituto Nacional de Estadística y Censos (INEC) en diciembre de 2015. Por su parte, las cifras de los indicadores socioeconómicos de la comunidad Compañía Lote Dos de la parroquia de Cangahua se calcularon del Censo de Condiciones de Vida de la Parroquia Cangahua, el mismo que fue levantado por docentes y estudiantes de la Facultad de Ciencias Económicas de la Universidad Central del Ecuador en el año 2015.

2 La información referente a la comunidad Compañía Lote Dos fue recopilada a través del diseño de grupos focales con la finalidad de conocer la historia y algunas características de la misma.

\section{REFERENCIAS}

Aguerre, C. (2011). Comunicación, stakeholders y las redes de confianza en las organizaciones. Dixit, (15), 34-38.

Atria, R., Siles, M., Arriagada, I., Robison, L. y Whiteford, S. (2003). Capital social y reducción de la pobreza en América Latina y el Caribe: en busca de un nuevo paradigma. Recuperado de http://repositorio.cepal.org/bitstream/handle/11362/2345/So29693.pdf?sequence=1

Baker, W. (1990, November). Market networks and corporate behavior. American Journal of Sociology, 96(3). 589-625. Recuperado de http://www.journals. uchicago.edu/doi/abs/10.1086/229573

Bourdieu, P. (1985). The forms of capital. In J. G. Richardson (Ed.), Handbook of theory and research for the sociology of education (pp. 241-258). New York, UsA: Greenwood Press.

Bourdieu, P. (1985, Mars 17). Le capital social. Actes de la recherche en science sociales, (10), 1-2. Recuperado de http://sepladerym.hidalgo.gob.mx/equidad/ medios/Libro9.pdf

Bourdieu, P. (1997). Razones prácticas sobre la teoría de la acción. Barcelona, España: Editorial Anagrama. 
Campbell, C y Jovchelovitch, S. (2000). Health, community and development: toward a social psychology of participation. Reis: Revista Española de investigaciones sociológicas, (10), 225-270.

Carpio, J. (2001). Desarrollo local en los espacios rurales. Polis, Revista de la Universidad Bolivariana, $1(2), 45-56$.

Coleman, J. (1988). Social capital in the creation of human capital. American Journal of Sociology, 94, 95120. Recuperado de http://www.jstor.org/stable/2780243

Coleman, J. (1990). The foundations of social theory. Recuperado de http://www.hup.harvard.edu/catalog.php?isbn= 9780674312265

Coleman, J. (february, 1993). The racional reconstruction of society. American Sociological Review, 58(1), 1-15. Recuperado de http://web.ics.purdue.edu/ hoganr/SOC\%20602/Spring\%202014/ Coleman\%201993.pdf

Delhey, J. and Newton K. (2002). Who Trusts? The Origins of Social Trust in Seven Nations. Berlin, Germany: Social Science Research Center Berlin.

Durston, J. (diciembre, 1999). El capital social. Revista de la CEPAL, (69), 103-108.

Durston, J. (julio, 2000). ¿Qué es el capital social comunitario? Serie Políticas Sociales de la Naciones Unidas, (33), 2-45. Recuperado de http://repositorio.cepal.org/ bitstream/handle/11362/5969/ Sooo7574_es.pdf?sequence $=1$

Falleto, E y Martner, G. (1986). Repensar el futuro, estilos de desarrollo. Compilación de ensayos. Caracas, Venezuela: Editorial Nueva Sociedad.

Fernández, P. (2012, enero 26). Pierre Bourdieu: El capital social. Homenaje de los sociologos peruanos al maestro, colega y amigo. Sociología Política. Recuperado de http://pabloraulfernandez.blogspot.com/2012/o1/el-capital-social-es-un-concepto-con.html

Forni, P., Castronuovo, L. y Nardone, M. (2009, julio 9). Redes, capital social y desarrollo comunitario. Una aproximaciòn teórico-metodológica. Análisis organizacional. Revista Latinoamericana de Ciencias Sociales, 1(1), 113-146.

Fukuyama, F. (2001). Social capital, civil society, and development. Third World Quarterly, 22(5), 47-61.

Gujarati, D. y Porter, D. (2010). Econometría. México DF, México: McGraw-Hill.

Gutiérrez, S. (2016). Capital Social, cultura organizacional, cultura innovadora y su incidencia en las organizaciones productivas rurales colaborativas. Revista Economía y Sociedad, (34), 119-136.

Ibañez, E., Ramírez de Haro, G. y Cuerdo, M. (2004). El capital social y el estudio del desarrollo económico. Madrid, España: Universidad Rey Juan Carlos.

Lesser, E. (2000). Knowledge and social capital. UsA: Global Real.

Loury, G. (june, 1977). A dynamic theory of racial income differences. In P. A. Wallace and A. LeMund (Eds.), Women, Minorities, and Employment Discrimination, (153-186). Lexington, USA: Lexington Books.

Martínez, J. (2010). Capital social y desarrollo económico en Puebla, México. Desarrollo local y cooperación internacional, (s. d.), 57-88.

Matus, C. (1986). Política, planificación y gobierno. Cepal. Recuperado de http://www.trabajosocial.unlp. edu.ar/uploads/docs/2_carlos_matus_politica_planificacion_y_gobierno_.pdf.

Mavares, A., Díaz, M., Colina, F., Lombardi, D. y Prieto, J. (1999). El capital humano, el capital social y su importancia para el desarrollo económico. Revista de Ciencias Sociales, 5(2), 129-136.

Nie, N., Junn, J. and Stehlik-Barry, K. (1996). Education and democratic citizenship in America. Chicago, usa: Chicago University Press. 
Organización de las Naciones Unidas para la Agricultura y la Alimentación (FAO). (2008). Organización comunitaria. Recuperado de http://www.fao.org/3/a-as496s.pdf

Portela, M. y Neira, I. (2002). Capital social: Concepto y estudio econométrico sobre el capital social en España. Estudios Económicos de Desarrollo Internacional, 2(2), (s. p.). Recuperado de https://www.researchgate.net/publication/230852702_Capital_Social_Concepto_y_Estudio_ Econometrico_sobre_el_Capital_Social_en_Espana

Portes, A. (1999). Capital social: sus orígenes y aplicaciones en la sociedad moderna. Siempro. Recuperado de http://sepladerym.hidalgo.gob.mx/equidad/medios/ Librog.pdf

Putnam, R. (1993). Making democracy work: civic traditions in modern Italy. Princeton, usA: Princeton University Press.

Putnam, R. (2001). Social Capital: Measurement and consequences isuma: Canadian. Journal of Policy Research, (34), 41-51.

Ramírez, J. (2005). Tres visiones sobre capital social: Bordieu, Coleman y Putnam. Acta Republicana, 4(4), 21-36. Recuperado de http://studylib.es/doc/6090730/ tres-visiones-sobre-capi

Roche, M. (2004). Social Policy and Social Capital: A Clear Case of Putting Merit before Method? Social Policy and Society, 3(2), 97-111.

Sánchez, V. (2013). El capital social como instrumento del análisis económico. Cuaderno de relaciones laborales, 31(2), 473-493.

Schiff, M. (April, 1992). Social capital, labor mobility and welfare. Ration, 4, 157-175. Recuperado de http:// journals.sagepub.com/doi/pdf/10.1177/104346319200400 2003

Stone, W. (2000). Social capital, social cohesion and social security. Journal of Economic Literature, (40), 175-154.

Temple, J. (2001, June 19). Growth effects of education and social capital in the oecd countries. OECD Economic Studies, 2(33). Recuperado de http://www.oecd. org/eco/growth/18452154.pdf

Van Deth, J. W. (2008). Measuring Social Capital. In D. Castiglione, J. W. Van Deth, and G. Wolleb (Eds.), The handbook of social capital. Oxford, uk. Oxford University Press.

Woolcock, M. (1998). Social capital and economic development: towards a theoretical synthesis and policy framework. Theory and Society, 27(2), 151-208. Recuperado de http://www.socialcapitalgateway.org/content/paper/woolcock-m-1998-social-capital-and-economic-development-towardtheoretical-synthesis-a

Wooldridge, J. M. (2010). Introducción a la econometría. Un enfoque moderno. México DF, México: Cengage Learning. 
